\title{
A Descriptive Study of Hematologic Characteristics of Malaria Patients Attending A Tertiary Care Hospital in The Region of Kutch
}

\author{
Nidhi N Shah and Riti T.K Sinha* \\ Department of Pathology, Gujarat Adani Institute of Medical Sciences, Bhuj, Gujarat, India.
}

\begin{abstract}
Background: In India, the epidemiology of malaria is complex because of geo-ecological diversity, multi-ethnicity, and wide distribution of nine anopheline vectors transmitting two commonest plasmodia species. Considering the fact, it is vital that every centre has its own demographic and pathological data about the profile of malaria patients in its catchment area.

Methods: The present retrospective study was carried in the Department of Pathology, Gujarat Adani Institute of Medical Sciences (GAIMS), Bhuj for a period of one year. A total of 102 cases were included in the study. The blood samples were collected in Ethylenediamine tetra-acetic acid (EDTA) vacutainers and smears were prepared. Leishman staining and field staining on thick smears was done according to the published protocols. The diagnosis of malaria parasite was confirmed by a positive peripheral smear examination. Rings, schizonts and gametocytes of the malaria parasites (plasmodium vivax and plasmodium falciparum) were viewed for diagnosis. Thrombocytopenia was considered when the platelet count was less than $150 \times 109 / \mathrm{L}$ and leucopenia when the total leucocyte count was less than 4,000 cells /cu mm.

Result: In our study, 102 malaria positive cases were investigated for platelet count and total leucocyte count. Out of the 102 cases, male population was affected more than female. Out of the various malaria species, plasmodium vivax was the most prevalent (82.35\%). The most common age group affected by various species varied, with age group of 1-10 years being most common for plasmodium falciparum cases, 21 to 30 years being most common for plasmodium vivax and 11 to 20 years being most common for mixed infection. All the cases of plasmodium falciparum were found to be with normal leucocyte count whereas in plasmodium vivax $82.14 \%$ cases were with normal leucocyte count and only $17.85 \%$ cases with leucopenia. Mixed infection had almost similar scenario with $71.42 \%$ cases with normal leucocyte count and $28.57 \%$ with leucopenia. In our study more number of cases of plasmodium falciparum was associated with thrombocytopenia (25\%) as compared to plasmodium vivax (11.9\%) and none in mixed infection.
\end{abstract}

Conclusion: The high prevalence of malaria suggests the importance of timely diagnosis . The early identification of thrombocytopenia and leucopenia aids in timely management. This helps to decrease complicated malaria cases and its related mortality.

\section{Keywords: Plasmodium Vivax, Plasmodium Falciparum, Leucopenia, Thrombocytopenia.}

\section{Introduction}

Malaria is one of the important infectious disease that is caused by malaria parasites of man -plasmodium vivax (PV), plasmodium falciparum (PF), plasmodium ovale and plasmodium malariae. It is transmitted by a single bite of the anopheles mosquito by sporozoites that it transmits via its bite. ${ }^{[1]}$

The worldwide estimation of malaria cases is 216 million with 4,45,000 deaths being attributed to it. It is estimated by World Health Organization that India accounts for $6 \%$ of the malaria cases world-wide. India has a vision of malaria free country by 2027 and elimination by 2030. ${ }^{[2]}$

Thrombocytopenia ${ }^{[3]}$, leucopenia ${ }^{[4]}$ and anaemia ${ }^{[5]}$ are common presentations of malaria infection. Bone marrow suppression along with haemo-phagocytosis is attributed to the cytopenias that occur in malaria infection. ${ }^{[5]}$ Erythrocytes destruction by plasmodia is responsible for anaemia in malaria. Increase in osmotic and mechanical fragility of parasitized red blood cells supports this observation. Inhibition of marrow activity may be an additional factor. ${ }^{[6]}$ Thrombocytopenia is one of the most common complications of malaria infestation along with leucopenia. ${ }^{[7]}$ The association of thrombocytopenia with $\mathrm{PF}$ is widely recognized, but such association with PV infection has also been assessed in the recent literature. ${ }^{\left[{ }^{[8}\right.}$ There is significant correlation between the hematological alteration in malaria and the clinical severity of disease, though the hematological alterations rarely cause mortality in the infected patients; its excess can lead to complications. Complications involve the nervous, respiratory, renal and/or hematopoietic systems. Black water fever is a complication of PF malaria. Early diagnosis is of paramount importance and malaria must be considered in every febrile patient with a history of fever and travel from an endemic region.

The present study was undertaken to study the prevalence of malaria species and their co-relation with thrombocytopenia and leucopenia. 


\section{Materials and Methods}

The present retrospective study was carried in the Department of Pathology, Gujarat Adani Institute of Medical Sciences (GAIMS), Bhuj for a period of one year. A total of 102 cases were included in the study. The blood samples were collected in Ethylenediamine tetra-acetic acid (EDTA) vacutainers and smears were prepared. Leishman staining and field staining on thick smears was done according to the published protocols. ${ }^{[9,10]}$ Other hematological parameters like complete blood count (CBC), was also performed by Beckman Coulter LH 750 series. The diagnosis of malaria parasite was confirmed by a positive peripheral smear examination. Rings and gametocytes of PF and rings and schizonts of PV were viewed for diagnosis. CBC was reassessed once the diagnosis of malaria was rendered. Thrombocytopenia was considered when the platelet count was less than 150 $\mathrm{x} 10^{9 / \mathrm{L}^{[11]}}$ and leucopenia when the total leucocyte count (TLC) was less than 4,000 cells/cumm. ${ }^{[12]}$

\section{Result}

Out of total 102 patients with malaria infection, male population was affected more than female. (Table 1). Most common age group affected was 21-30 years in males, 11-
20 years in females. (Table 1). PV was the most common malaria parasite $(82.35 \%)$, followed by mixed infection $(13.73 \%)$. (Table 2). PV was most common in the age group of 21-30 years. (Table 2). PF was most common in the age group of 1-10 years. (Table 2). Mixed malaria infection was most common in 11-20 years age group. (Table 2)

The most common age group affected in all the malaria species was 21-30 years accounting for $27.45 \%$ of cases. (Table 2). In PV, majority of cases $82.15 \%$ were found with normal leucocyte count and $17.85 \%$ were found with leucopenia. (Table 3) (Figure 1). In mixed infection, majority of cases $71.43 \%$ were found to have normal leucocyte count and $28.57 \%$ were found with leucopenia. (Table 3). In PF all the cases had normal TLC. (Table 3). $88.1 \%$ of PV had normal platelet count while $11.9 \%$ had thrombocytopenia. (Table 3). All cases of mixed infection presented with normal platelet count. (Table 3). $75 \%$ of PF cases had normal platelet count and $25 \%$ had thrombocytopenia. (Table 3) (Figure 2)

\section{Discussion}

Plasmodium infections are a major public health problem, resulting in millions of deaths annually worldwide. Although $\mathrm{PF}$ is responsible for the majority of severe complications

List of abbreviations -

- Plasmodium Vivax - PV

- Plasmodium Falciparum - PF

- $\quad$ Percentage - \%

- Number $-\mathrm{N}$

- Total leucocyte count - TLC

Table 1: Gender distribution according to different age groups.

\begin{tabular}{|c|c|c|c|}
\hline Age group & Male & Female & Total \\
\hline$<1$ & 1 & 0 & 1 \\
\hline 1 to 10 & 7 & 8 & 21 \\
\hline 11 to 20 & 11 & 2 & 28 \\
\hline 21 to 30 & 26 & 6 & 15 \\
\hline 31 to 40 & 9 & 2 & 11 \\
\hline 41 to 50 & 9 & 2 & 5 \\
\hline 61 to 60 & 3 & 2 & 6 \\
\hline
\end{tabular}

Table 2: Species distribution along with total percentage in each age group.

\begin{tabular}{|c|c|c|c|c|c|}
\hline Age group & PF & PV & Mixed & Total & Percentage (\%) \\
\hline$<1$ & - & 1 & - & 1 & 1.02 \\
\hline 1 to 10 & 2 & 11 & 4 & 21 & 14.71 \\
\hline 11 to 20 & 1 & 16 & 3 & 28 & 20.59 \\
\hline 21 to 30 & - & 25 & 2 & 15 & 14.45 \\
\hline 31 to 40 & - & 13 & & & 14.71 \\
\hline
\end{tabular}




\begin{tabular}{|c|c|c|c|c|c|}
\hline Age group & PF & PV & Mixed & Total & Percentage (\%) \\
\hline 41 to 50 & - & 10 & 1 & 5 & 10.78 \\
\hline 51 to 60 & - & 5 & - & 6 & 4.9 \\
\hline 61 to 70 & 1 & 3 & $14(13.73 \%)$ & 102 & 5.84 \\
\hline Total & $4(3.92 \%)$ & $84(82.35 \%)$ & 100 \\
\hline
\end{tabular}

Table 3: Relation between malaria type and TLC.

\begin{tabular}{|l|l|l|l|l|}
\hline Malaria type (number) & Leucopenia (\%) & Normal TLC (\%) & Thrombocytopenia (\%) & Normal (\%) \\
\hline PF (4) & 0 & $4(100 \%)$ & $1(25 \%)$ & $3(75 \%)$ \\
\hline PV (84) & $15(17.85 \%)$ & $69(82.15 \%)$ & $10(11.9 \%)$ & $74(88.1 \%)$ \\
\hline Mixed infection (14) & $4(28.57 \%)$ & $10(71.43 \%)$ & 0 & $14(100 \%)$ \\
\hline Total & $\mathbf{1 9 ( 1 8 . 6 3 \% )}$ & $\mathbf{8 3 ( 8 1 . 3 7 \% )}$ & $\mathbf{1 1 ( 1 0 . 7 \% )}$ & $\mathbf{9 1 ( 8 9 . 3 \% )}$ \\
\hline
\end{tabular}

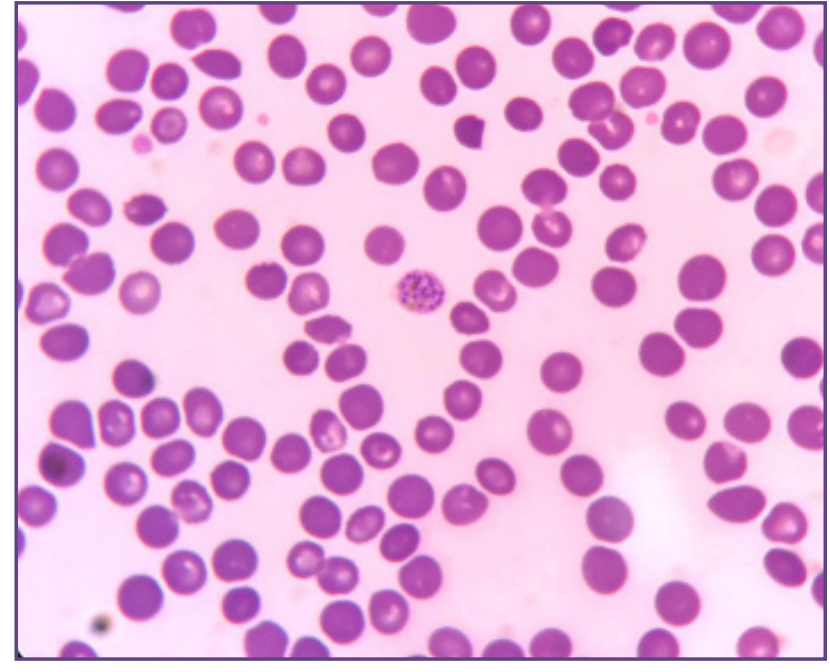

Fig. 1: Schizonts of plasmodium vivax along with leucopenia and thrombocytopenia. (Leishman stain, 1000X).

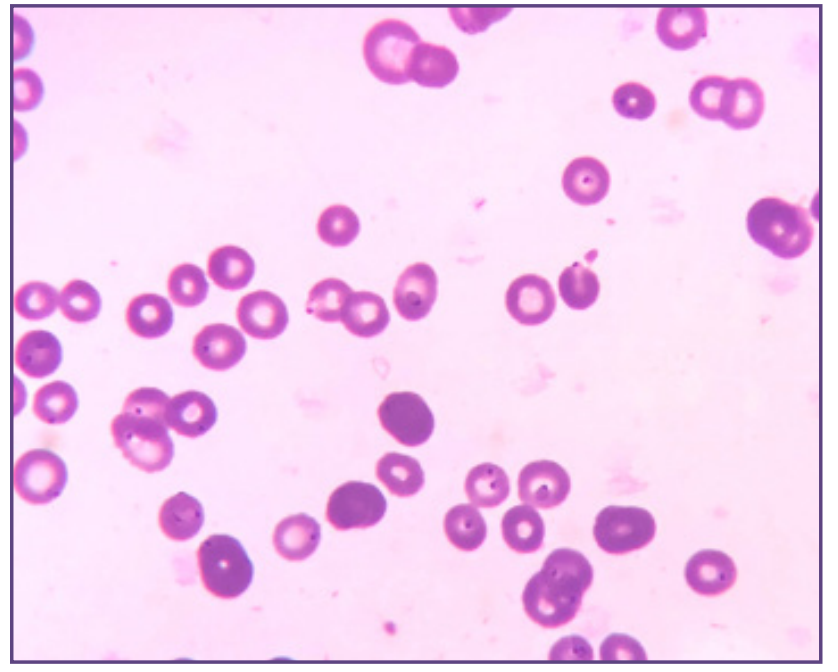

Fig. 2: Multiple ring forms of plasmodium falciparum along with thrombocytopenia. (Leishman stain, 1000X).

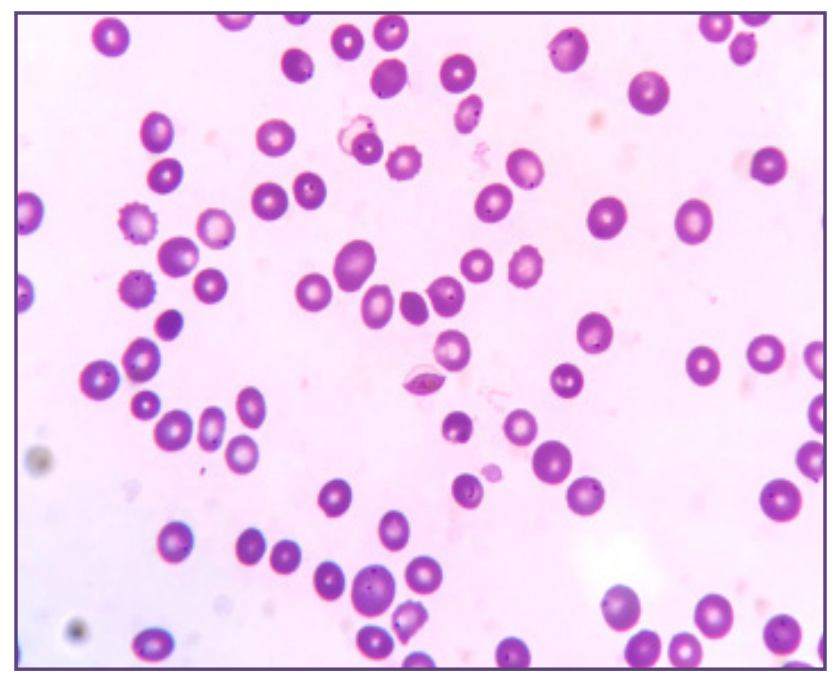

Fig. 3: Gametocyte and multiple ring forms of plasmodium falciparum with thrombocytopenia and leucopenia. (Leishman stain, 1000X). 
and malaria-associated mortality; vivax malaria has now clearly emerged as a potentially lethal condition. ${ }^{[13-14]}$ Malaria is endemic in many parts of the world including India, with $\mathrm{PV}$ being the most common species worldwide. ${ }^{[15]}$ Malaria (from the Italian mal'aria, meaning bad air) is an acute and sometimes chronic infection of the blood stream characterized by fever, anaemia and splenomegaly. It is caused by apicomplexan parasite of the genus Plasmodium. The defining clinical features of a malaria attack or paroxysm consists of, in order, shaking chills, fever (up to 40 degree celcius or higher) and generalized diaphoresis, followed by resolution of fever. The paroxysm occurs over 6 to 10 hours and is initiated by the synchronous rupture of erythrocytes with the release of new infectious blood stage form known as merozoites. ${ }^{[6]}$ The life cycle of the malarial parasite is complex and multistage process of 8-21 days consisting of two stages - sexual phase and asexual stage. The sexual phase or sporogony occurs within the mosquitos. When the female anopheles mosquito draws blood from an individual infected with malaria, the gametocyte of the parasite finds their way into the gut of the mosquito where the sporogonic cycle is initiated. The male and female gametes fuse to form zygotes which subsequently develop later into ookinetes which develop into oocytes. Growth and divison of each oocyte produce thousands of active haploid forms called sporozoites which are released into the body cavity of mosquito from where they travel and reach the salivary glands. When mosquito thus loaded with sporozoites takes another blood meal the spoorozoites are injected into the human blood stream. In humans the schizogony that is the asexual phase occurs first in liver and then in the erythrocyte. The sporozoites that find the blood vessel, reach the liver within a few hours. The sporozoites negotiate through the liver sinusoids and reach the hepatocytes where they develop into a schizont containing many merozoites. These merozoites are gradually released into the blood stream and initiate the erythrocytic schizogony. Within the erythrocyte repeated cycles of parasitic development occurs and hundreds of fresh daughter parasites are released that invade more erythrocytes. A small proportion of asexual parasites do not undergo schizogony but differentiate into the sexual stage gametocytes. These gametocytes are extracellular and non pathogenic and help to transfer the infection. ${ }^{[6]}$

Laboratory evaluation of patients suspected of having malaria along with card tests, continues to rely on the gold standard method of microscopic examination of thick and thin blood films to demonstrate the different stages of parasites. Schizonts are present in smear of PV (Figure 1). Ring forms and multiple rings within a single RBC, a characteristic feature of PF is also seen (Figure 2). The gametocyte stage is also seen in PF (Figure 3). The recent development of immunocapture assays for the detection of plasmodium-specific lactate dehydrogenase appears to provide a high degree of sensitivity and specificity in the diagnosis of malaria. One of these, tests configured as a 'dipstick' method, is especially promising in situations where ease of performance is critical and usual laboratory facilities are lacking. More advanced laboratory methods, including acridine orange staining or detection of parasitespecific DNA provide enhanced sensitivity and specificity but are generally not appropriate or available for smaller laboratories. Erythrocytes destruction by plasmodia is responsible for the anaemia in malaria. Osmotic and mechanical fragility of parasitized erythrocyte is increased, thus supporting this observation. Inhibition of marrow activity may be an additional factor. Blackwater fever is a complication of PF malaria. ${ }^{[6]}$ Thrombocytopenia and leucopenia in patients with malaria is a frequent finding at health care facilities, but not much is known about prevalence of these in the people of Kutch. In this study $\mathrm{PV}$ was found to be the most prevalent species followed by mixed infection and PF. Thrombocytopenia is defined as reduction in the peripheral blood platelet count below the lower normal limit of $150 \times 10^{9} / \mathrm{L}^{[11]}$ Although the mechanism of thrombocytopenia in malaria is not clear , possibility of patho-immunological mechanisms such as platelet phagocytosis, immunoglobulin $\mathrm{G}$ binding to the platelet bound malaria antigens with sequestration of engulfed platelet in spleen - phagocytosis by spleenic macrophages, macrophage colony stimulating factor, spleenic pooling and oxidative stress leading these to excessive removal of platelets or their excessive destruction has been suggested. Though thrombocytopenia is commonly reported in malaria cases, its mechanism are poorly understood. ${ }^{[16]}$ Thrombocytopenia was found in $62.9 \%$ cases of PV by Coelho HCC et $\mathrm{a}^{[16]}$ as compared to $11.9 \%$ in our present study. The cause of thrombocytopenia was platelet phagocytosis. They also found that phagocytosis index was higher in thrombocytopenic patients compared to non thrombocytopenic patients. There was no difference in platelet activation. Interleukin (IL) 6, IL 10 and tumor necrosis factor gamma (TNF) values were higher in thrombocytopenia with a positive co relation is found between TNF alpha and phagocytosis. ${ }^{[16]}$ High frequency of thrombocytopenia in patients with malaria has been well-documented in several studies. ${ }^{[17-20]}$ Kochar et $\mathrm{al}^{[17]}$ have recently shown that severe thrombocytopenia (platelet count $<20 \times 10^{3} / \mathrm{mm}^{3}$ ) is a common manifestation in patients with vivax mono-infection confirmed by PCR. ${ }^{[17,18]}$ Our study reports thrombocytopenia in $10.7 \%$ of the total cases (Table 3) (Figure 1), which is in contrast to the $78 \%$ reported by Gupta $\mathrm{N}$ et al ${ }^{[19]}, 72 \%$ by Colonel et al ${ }^{[20]}$ 
In our study cases of thrombocytopenia were associated with both PV and PF infection.

It has been found that TLC are generally lower in malaria patients compared to healthy patients. Several theories attempt to explain these changes: bone marrow suppression secondary to immune response imbalances, lower average cell life and leucocyte redistribution to lymphoid organs and tissue with increased inflammatory response or sequestration in microvasculature. ${ }^{[21-25]}$ Leucopenia is defined as total leucocyte count less than 4,000 cell/cumm. In our study leucopenia was found to be more associated with malaria infection than thrombocytopenia. We report leucopenia in $18.6 \%$ of the total cases (Table 3) (Figure 2 ), which is in concordance to the various other studies carried out in endemic regions like $24.6 \%$ by Reshma Kini et $\mathrm{a}^{[26]}, 11 \%$ by Abro et $\mathrm{a}^{[27]}, 13.3 \%$ by Bashwari et $\mathrm{a} \mathrm{l}^{[28]}$ and $10.2 \%$ by Ladani et al ${ }^{[29]}$.

Out of the total patients one patient was pregnant female in the age group of 21-30 years with mixed malaria infection. $\mathrm{PV}$ is the most common form observed in pregnancy. PV causes mild maternal anaemia and low birth weight infants, especially in multigravida women. PF malaria preferentially and adversely affects the placenta especially primi gravida. It is most severe, causing low birth weight infants and decreased survival. Along with RBC infestation plasmodia appears to adhere to chorionic villi, increases inflammation and induces placental necrosis. Diagnosis of sporadic cases usually depends on maternal blood film examination. ${ }^{[6]}$

\section{Conclusion}

The high prevalence of malaria suggests the importance of timely diagnosis and the timely identification of thrombocytopenia and leucopenia aiding timely management. This helps to decrease complicated malaria cases and related mortality. To the best of our knowledge this study, in this region has been done for the first time.

\section{Acknowledgements}

To all our patients.

\section{Reference}

1. Chatterjee K D. Class sporozoa. Parasitology, Protozoology and Helminthology. Fifth edition. Calcutta: Shree Saraswati Press Ltd; 1965:57-84.

2. World malaria report 2017.Geneva: World Health Organization; 2017.

3. Horstmann R, Dietrich M, Bienzle U, Rasche H. Malariainduced thrombocytopenia. Ann Hematol.1981;42:157-64.

4. McKenzie FE, Prudhomme W, Magill A, et al. White blood cell counts and malaria. J Infect Dis.2005;192:323-30.
5. Menendez C, Fleming A, Alonso P. Malaria-related anaemia. Parasitol Today.2000;16:469-76.

6. Thomas R, Fritsche, James S. Medical Parasitology. In: Henry J, editor. Clinical Diagnosis and Management by Laboratory Methods. Twentieth edition. Noida:Saurabh Print-O-Pack;2001:1196-1240.

7. Gupta NK, Bansal SB, Jain UC, Sahare K. Study of thrombocytopenia in patients of malaria. Tropical Parasitology.2013;3:58-61.

8. Cho Naing, Maxine AW. Severe thrombocytopaenia in patients with vivax malaria compared to falciparum malaria: a systematic review and meta-analysis. Infectious Diseases of Poverty.2018;8:7-10.

9. Field JW, Sandosham AA. The Romanowsky stains-aqueous or methanolic?. Transactions of the Royal Society of Tropical Medicine and Hygiene. 1964;35:164-72.

10. Field JW. The morphology of malaria parasites in thick blood films. Part IV. The identification of species and phase. Transactions of the Royal Society of Tropical Medicine and Hygiene.1941;34:405-14.

11. De Gruchy GC. The Haemorrhagic Disorders;Capillary and Platelet defects. In: Frank F, Colin C, David P, Bryan R, editors. de Gruchy's Clinical Haematology in Medical Practice. Fifth edition. Noida:Sheel Print-OPack;2008:360-405.

12. Hoffbrand AV et al. Postgraduate hematology. Sixth edition. Hoboken:Black-well Publishing Ltd.;2011.

13. Anstey NM, Russell B, Yeo TW, Price RN. The pathophysiology of vivax malaria. Trends Parasitol.2009;25:220-227.

14. Lacerda M, Mourao M, Alexandre M, et al. Understanding the clinical spectrum of complicated Plasmodium vivax malaria: a systematic review on the contributions of the Brazilian literature. Malar J.2012;11:12.

15. WHO. Global technical strategy for malaria 2016-2030. Geneva: World Health Organization;2015.

16. Coelho H, Lopes S, Pimentel J, et al. Thrombocytopenia in Plasmodium vivax Malaria Is Related to Platelets Phagocytosis. PLoS ONE.2013;8:e63410.

17. Kochar DK, Das A, Kochar A, et al. Thrombocytopenia in Plasmodium falciparum, Plasmodium vivax and mixed infection malaria: a study from Bikaner (Northwestern India). Platelets.2010;21:623-7.

18. Tanwar GS, Khatri PC, Chahar CK, et al. Thrombocytopenia in childhood malaria with special reference to P. vivax monoinfection: A study from Bikaner (Northwestern India). Platelets.2012;23:211-6.

19. Gupta NK, Bansal SB, Jain UC, Sahare K. Study of thrombocytopenia in patients of malaria. Tropical Parasitology.2013;3:58-61.

20. Colonel KM, Bhika RD, Khalid S, Khalique-ur-Rehman S, Syes ZA. Severe thrombocytopenia and prolonged bleeding 
time in patients with malaria (a clinical study of 162 malaria cases). World Appl Sci J.2010;9:484-8.

21. McKenzie F, Prudhomme W, Magill A,et al. White blood cell counts and malaria. Journal of Infectious Diseases.2005;192:323-30.

22. Wickramasinghe S, Abdalla S. Blood and bonemarrow changes in malaria. Baillieres Best Practice and Research in Clinical Haematology.2000;13:277-99.

23. DeMast Q, Sweep F, McCall M, et al. A decrease of plasma macrophage migration inhibitory factor concentration is associated with lower numbers of circulating lymphocytes in experimental Plasmodium falciparum malaria. Parasite Immunology. 2008;30:133-8.

24. Helmby H, J"onsson G, Troye-Blomberg M. Cellular changes and apoptosis in the spleens and peripheral blood of mice infected with blood-stage Plasmodium chabaudi chabaudi AS. Infection and Immunity.2000;68:1485-90.
25. Mohan K,Stevenson M. Dyserythropoiesis and severe anaemia associated with malaria correlate with deficient interleukin-12 production. British Journal of Haematology. 1998;103:942-949.

26. Kini RG, Chandrashekhar J. Parasite and the Circulating Pool- Characterisation of Leukocyte Number and Morphology in Malaria. Journal of Clinical and Diagnostic Research : JCDR.2016;10:44-8.

27. Abro AH,Ustadi AM,Younis NJ,Abdou AS,HamedDA. Malaria and hematological changes. Pak J Med Sci.2008;24:287-91.

28. Bashwari LAM,MAndil AA,Bhanassy AA,Hamed DA,Saleh AA. Malaria: haematological aspects. Annals of Saudi Medicine.2002;22:372-7.

29. Ladhani S,Lowe B,Cole AO,Kowuondo K,Newton CR. Changes in WBCs and Platelets in children in falciparum malaria: relationship to disease outcome. Br J Haematol. 2002;19:839-49.

*Corresponding author:

Dr. Riti T.K Sinha, Associate Professor, Department of Pathology, Gujarat Adani Institute of Medical Sciences, Bhuj, Gujarat, India. 370001

Phone: +91 9641429455

Email: rsinha75@gmail.com

Financial or other Competing Interests: None. 